\title{
Symptomatic loosening of a total knee arthroplasty caused by a tibial chondrosarcoma - a case report
}

\author{
Jakob T Sieker, Maximilian Rudert and Andre F Steinert ${ }^{*}$
}

\begin{abstract}
Premature implant loosening following total knee arthroplasty (TKA) can have several causes. In this article we report on a rare case of a 74 year old male patient suffering tibial component loosening 14 month after primary TKA. The patient did neither have any malignancies nor joint arthroplasty before. Upon clinical examination the range of motion in the diseased knee was painfully restricted to $80^{\circ}$ of knee flexion, with the patient increasingly suffering sleeping and resting pain, and also at weight bearing. In standard radiographs, loosening of the TKA due to a large osteolysis at the tibial component was evident. Local computed tomography (CT) of the right knee revealed loosening of the tibial component due to a presumably malign bone tumor. For determination of the final diagnosis a representative biopsy of the tumor was taken by open surgery prior to the tumor resection.

Histopathologic evaluation of the biopsy revealed a periprosthetic myxoid chondrosarcoma of the proximal tibia. Pre-operative staging examination included CT scans of lung and abdomen, as well as a bone scintigraphy which revealed no signs of tumor metastasis in the body. Surgical management comprised wide tumor resection and implantation of a hinged tumor knee arthroplasty with replacements of the distal femur and proximal tibia, as well as a patella tendon replacement using a synthetic ligament. Revision surgery was necessary twice due to impaired wound healing and critical soft tissue coverage, and treatment included a gastrocnemius muscle flap with skin mesh graft covering. Unfortunately long-term follow-up examinations could not be obtained, as the patient deceased due to an alveolitis during rehabilitation. In summary, the specifics of this rare case of aseptic TKA loosening, and the unusual circumstances of chondrosarcoma diagnosis and treatment are informative for those providing surgical treatment of similar cases.
\end{abstract}

Keywords: Total knee arthroplasty; Bone tumor; Chondrosarcoma; Aseptic loosening

\section{Introduction}

Chondrosarcoma is an invasive musculoskeletal neoplasm, which is assumed to originate from malign transformed mesenchymal precursor cells, capable of forming a collagenous matrix (Aigner 2002). According to an analysis from the SEER (Surveillance, Epidemiology and End Results) Database the estimated annual incidence is $1 /$ 200000 with a mean age of 55 years (Giuffrida et al. 2009). Most frequently chondrosarcoma is located in the appendicular (44.5\%) or axial skeleton (31.1\%) (Giuffrida et al. 2009). The usual presentation of bone malignancies includes pain, due to destruction of the bone architecture, swelling and occasionally compression of surrounding

\footnotetext{
* Correspondence: a-steinert.klh@uni-wuerzburg.de
Department of Orthopaedic Surgery, König-Ludwig-Haus,

* Correspondence: a-steinert.klh@uni-wuerzburg.de
Department of Orthopaedic Surgery, König-Ludwig-Haus,

Julius-Maximilians-University Würzburg, Brettreichstr. 11, Würzburg D-97074, Germany
}

structures. When bone malignancy is suspected from clinical presentation and supported by radiographic imaging, referral to a specialized interdisciplinary center for further imaging and biopsy according to the American Joint Committee on Cancer (AJCC) guidelines is recommended (Ofluoglu et al. 2010; Edge et al. 2009).

When specified, the most common histological subtype is the myxoid chondrosarcoma (56\%), followed by the mesenchymal (25\%), dedifferentiated (10\%), juxtacortical (4\%), malignant chondroblastoma (4\%) and clear cell subtype (2\%) (Giuffrida et al. 2009). The survival rates differ between histological subtypes and grading, with a thirty year survival rate of low-grade chondrosarcomas (G1 and G2) being 76\%, compared to $50 \%$ in high-grade tumors (G3 and G4) (Giuffrida et al. 2009).

The predominant treatment of chondrosarcoma is surgical, with the status of the resection margins (R0, R1 or \\ Springer}


R2) being related with the rate of local recurrence (Giuffrida et al. 2009; Goda et al. 2011). Though skeletal and non-skeletal chondrosarcomas are described to have a high radiation- and chemotherapy-resistance (Onishi et al. 2011; Moussavi-Harami et al. 2006), clinical trials with successful radiation-treatment (Drilon et al. 2008; Rutz et al. 2008) or case-reports with promising chemotherapy application (Drilon et al. 2008; Dallas et al. 2012; Han et al. 2010) do exist. Here we report the diagnostic and surgical management, as well as the short-term outcome of a 76-year-old patient with tibial chondrosarcoma presenting with symptoms of prosthetic loosening 14 month after primary TKA.

\section{Case report}

The patient presented himself in our outpatient clinic 14 month after primary TKA which was implanted due to symptomatic knee osteoarthritis (OA) elsewhere. The pre-operative radiographs with anterior-posterior (ap.) and lateral view of the right knee are shown (Figure 1a and b). After an initial symptom-relief, stress-dependent pain reoccurred few months after the surgery. After a phase of stable symptoms, exacerbation occurred approximately 12 months postoperatively, including resting and sleeping pain, which triggered the referral to our outpatient clinic with suspicion of prosthetic loosening.

In the clinical presentation a painful swelling at the proximal tibia was palpable without signs of inflammation, knee ligaments were stable, while flexion was limited to $80^{\circ}$ due to pain. The obtained radiographs with anteriorposterior (ap.) and lateral view of the right knee (Figure 1c and d) showed a suspect Lodwick-grade II osteolytic lesion of the proximal tibia extending from the central epiand metaphysis to the anterior and lateral cortical bone. The interface to the intact bone was wide and blurred, the cortical bone showed discontinuation and complex reactions including spiculae (Figure 1c and d), which was confirmed by a local CT-scan (Figure 1e and f). In suspicion of bone malignancy, an open incision biopsy was performed that exhibited a whitish tumor mass with myxoid parts (Figure 1g). Histopathological evaluation revealed the diagnosis of a G2 myxoid chondrosarcoma, without signs of bacterial infection.

Staging was then completed and the case was discussed in the local interdisciplinary tumor board. In summary a local disease stage, without infiltration of popliteal structures or metastases was present, and a wide resection of the tumor with inclusion of the knee prosthesis was indicated according to the disease stage. Since limb-preserving resection and reconstruction is technically tedious and carries the risk of incomplete resection and local recurrence, amputation was also discussed with the patient and his family. In consideration of all pros and cons of the respective approaches, the patient wished to undergo wide tumor resection and reconstruction with a distal femur and proximal tibia

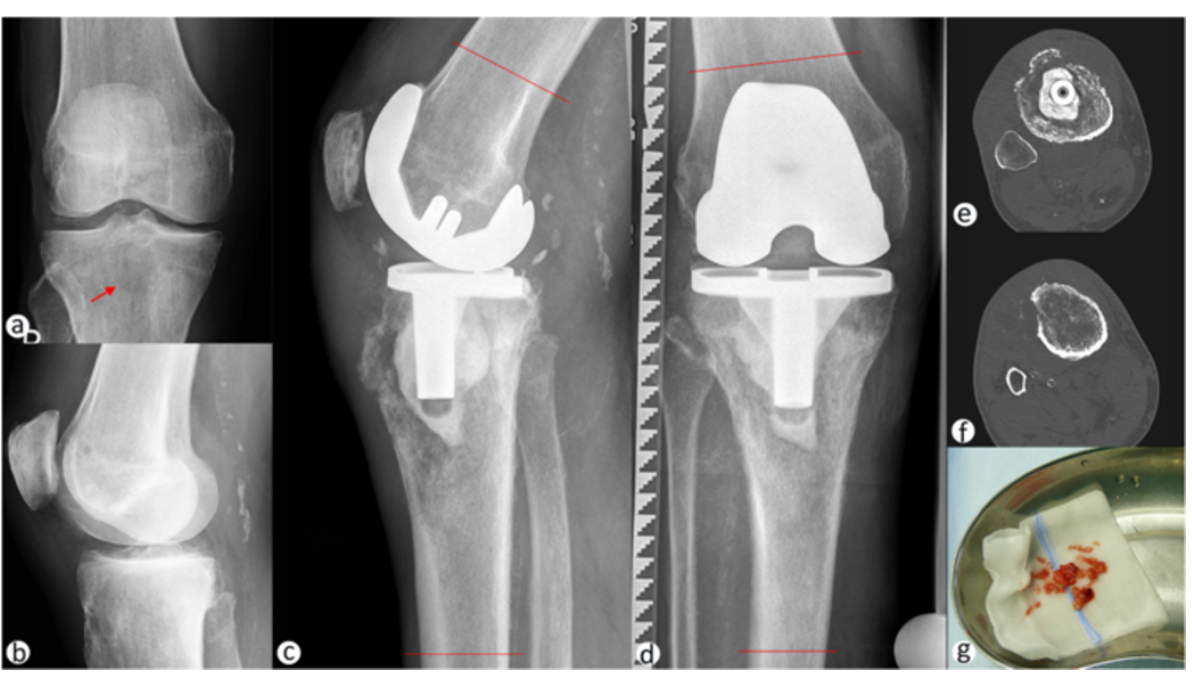

Figure 1 Implant loosening due to periendoprosthetic tibial chondrosarcoma. Anterior-posterior (a) and lateral (b) radiograph prior to total knee arthroplasty (TKA) depicting medial joint space narrowing and meniscal calcification. A radiolucent lesion in the central tibial plateau is indicated by a red arrow. Lateral (c) and anterior-posterior radiograph (d) 14 month after TKA surgery showing the osteolytic lesion in the proximal tibia, extending through the epi- and metaphyses from the central region to the lateral and anterior cortical bone. Permeation of the cortical bone with discontinuing periosteal reactions, including spiculae formation, can be observed. Preoperative planning was performed to determine plains of resection at $8 \mathrm{~cm}$ proximally and $15 \mathrm{~cm}$ distally to the joint line (indicated by red lines in c and d). Local CT-scans are showing cortical bone permeation, especially in the anterior and lateral aspects of the proximal tibia at the level of the cemented tibial implant (e) and below (f). Open incision biopsy was performed and multiple soft and bone tissue samples were obtained (g) and sent for microbiological and histopathological examination. 
replacing hinged knee prosthesis. This was then planned using the radiographs and CT-scans (Figure 1c-f), with the projected resections being located $8 \mathrm{~cm}$ proximal of the joint line at the femur and $15 \mathrm{~cm}$ distally of the joint line at the tibia. For the reconstruction the MML tumor knee system for distal femoral and proximal tibial replacement (Modular endoprosthetic system Munich-Lübeck, Orthodynamics GmbH, Lübeck, Germany) was chosen (Salis-Soglio et al. 2010), with an alloplastic reconstruction of the extensor mechanism (Holzapfel et al. 2011).

Resection of the knee joint with proximal tibia: Resection steps included scar excision, resection of the inner part of the patella with complete patellar-tendon (Figure 2a), resection of the joint capsule under preservation of the muscle-insertions of the pes-anserinus (Figure 2a) and the biceps-femoris tendons. Resection was initially performed as previously planned as soft tissue structures were without signs of tumor infiltration. After en-bloc resection the distal marrow showed suspicious tissue, therefore the intraoperative decision to extent the resection $5 \mathrm{~cm}$ distally was made (Figure 2b), which resulted in macro- and microscopically tumor free margins. The femoral resection was performed as planned $8 \mathrm{~cm}$ proximal of the joint line to match the planned implant, while no suspect bone or soft tissue changes were observed at the resection margins (Figure 2c). All resected tissues were sent in for histopathological examination. For reconstruction medullary canals were prepared followed by implantaion of trial-components for joint-line resonstruction, resulting in full extension and flexion without instability. Thorough irrigation was performed between steps and prior to cement fixation of the original components in the previously tested sizes. After reposition and connection, the previously detached tendons were reattached using FiberWire ${ }^{\bullet}$ sutures (Arthrex, Naples, FL, USA), with reconstruction of the patellar tendon being performed using a Trevira synthetic ligament (Telos, Marburg, Germany) (Figure 2d-f), before tension free wound closure was performed. After surgery full weightbearing with an extension brace and continuous passive motion was allowed.

The final histopathological examination revealed a pT2 pN0 L0 V0 tumor stage, with proof of the prior G2 grading. Resection status was proven as R0. While the

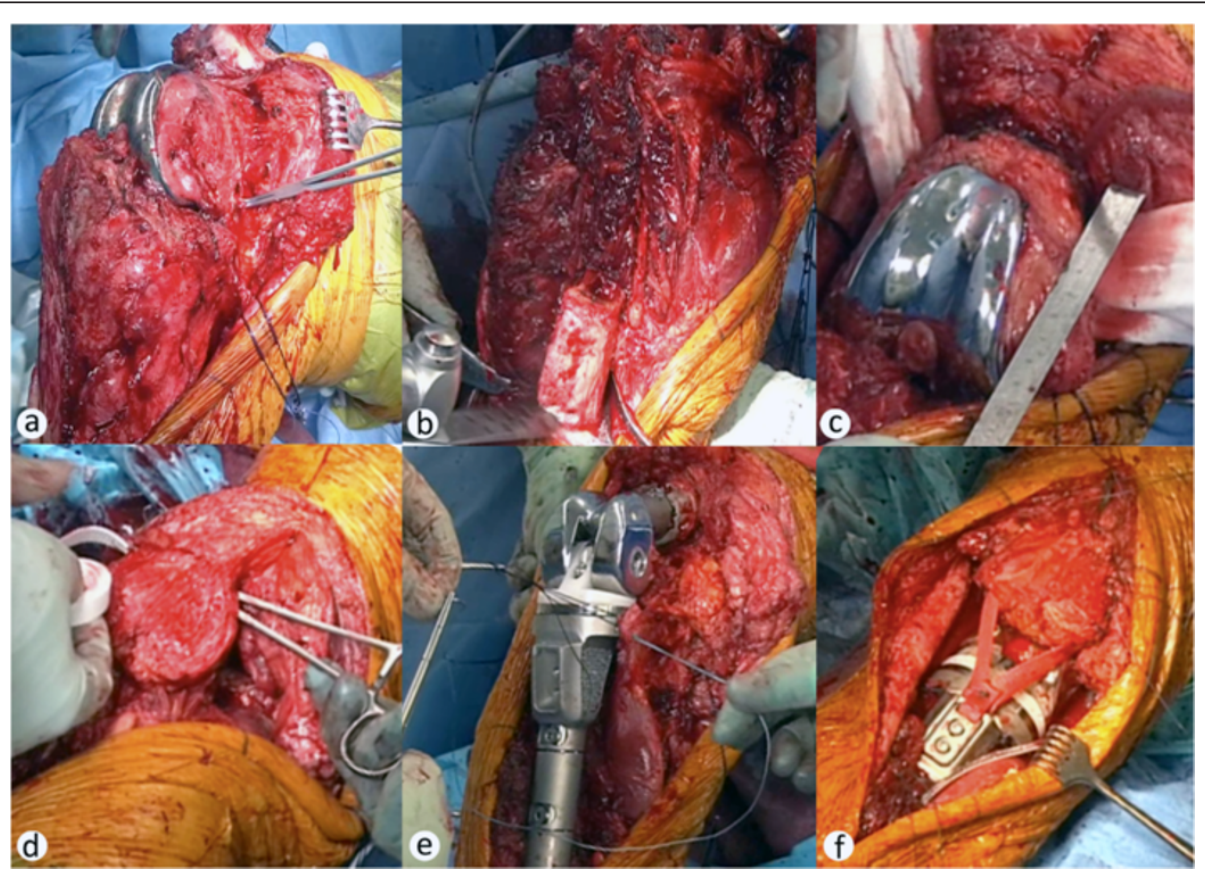

Figure 2 Surgical management of periendoprosthetic tibial chondrosarcoma. A medial parapatellar approach was used for exposition, while the patellar-ligament was cut proximally, because of macroscopic suspected tumor infiltration (a). Medial subcutaneous preparation was performed carefully and pes-anserinus muscle insertions were secured for later reattachment (a). After the tibia was exposed $15 \mathrm{~cm}$ from joint line, tibial resection was performed as planned and detachment from the popliteal neurovascular structures was performed carefully, allowing en-bloc tumor resection. Because of suspicious marrow tissue a second tibial resection was performed $5 \mathrm{~cm}$ more distally (b), which resulted in R0 resection status. The femoral cut was performed as planned at $8 \mathrm{~cm}$ proximal to the joint line, to include the femoral component and match the planned femoral component of the revision implant (c). After marrow preparations and trialing, reconstruction of the extensor mechanism was prepared by creating a suprapatellar tunnel in the quadriceps tendon and implantation of an alloplastic ligament (d). Original components were cemented, using the prior tested sizes. Pes-anserinus and biceps-femoris tendons were reattached at the correspondent implant sites (e). The alloplastic ligament was secured with the screw-mechanism of the tibial implant and additional sutures (f). Drains were implanted distant to the tumor location and tension-free wound closure was performed (f). 
postoperative radiographs (Figure 3a, b) showed correct implant positioning without signs of loosening or fracture, mobilization needed to be restricted due to delayed woundhealing over the site of patellar ligament-reconstruction, due to absent soft-tissue covering. This made operative wound revision necessary, including debridement (Figure 3c), gastrocnemius-flap-reconstruction (Figure $3 \mathrm{~d}$ ) in combination with a mesh-graft (Figure 3e) from the opposite thigh necessary. Thereafter he patient was mobile on the ward stage and healing of the graft was observed, as the patient could be discharged and transferred to a stationary rehabilitation department. Although all intraoperative cultures were sterile, antibiotic prophylaxis was continued orally during rehabilitation. Unfortunately - after initial progress in terms of mobility - the patient developed a progressive respiratory insufficiency due to an alveolitits, and deceased finally after a 4 week course of ICU care. Written consent was obtained from the patient's wife to publish data and pictures related to the treatment of her husband.

\section{Discussion}

The described case presented with symptomatic loosening 14 months after TKA. Although reports of implantrelated sarcoma formation do exist, in these the minimal observed interval from implantation to sarcoma diagnosis was 2.5 years (Keel et al. 2001). Since our case presented with an extensive tumor size early after TKA and an osteolytic lesion was described in the protocol of the externally performed surgery, we suspect that the chondrosarcoma was present during initial surgery. However, a biopsy, obtained from the osteolytic lesion during primary TKA remained inconclusive. Towards the management of patients with an extensive osteolytic lesion in combination with $\mathrm{OA}$, we recommend the open-incision biopsy prior to TKA and the evaluation in a pathological institute with expertise in musculoskeletal malignancies.

Towards the decision between amputation and prosthetic reconstruction, the local tumor extension, especially in relation to the adjacent nerves and vasculature, is important. In the presented case, the vasculature sparing $\mathrm{R} 0$ resection was possible and the limb-preservation using a proximal tibia replacing knee prosthesis was performed according to the patient's wish. For resection, a surgical approach through the patella bone was chosen, comprising the whole knee capsule, patellar and quadriceps tendon, followed by alloplastic reconstruction. Though technically feasible, this procedure obviously carries the risk of soft tissue loss, predisposing for wound-healing problems above the insertion site of the alloplastic graft. In our case, a primary soft tissue coverage using a gastrocnemiusflap-graft maybe would have enabled for faster wound healing and recovery. Therefore, soft tissue muscle flaps should be readily indicated and performed, when soft tissue coverage of large metal implants appears critical or insufficient.

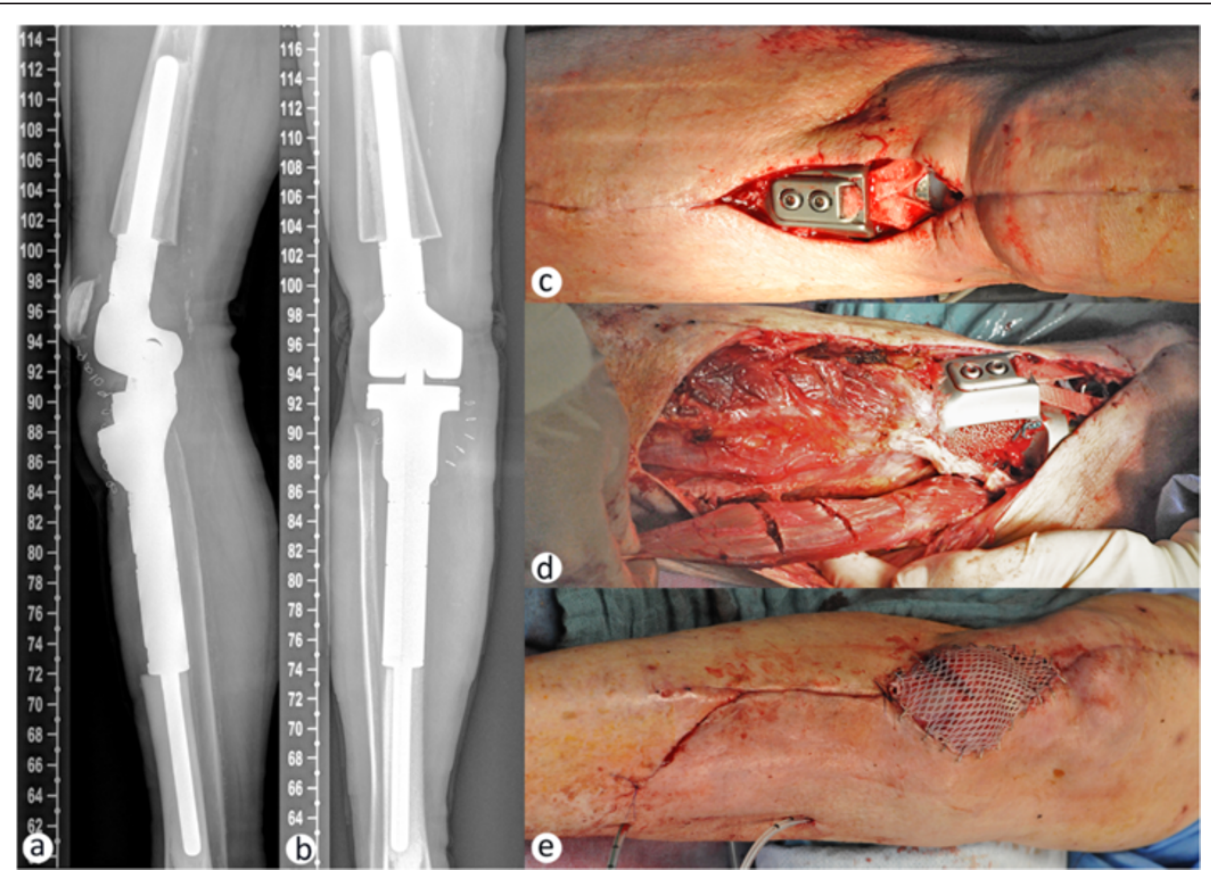

Figure 3 Soft tissue coverage following megaendoprosthetic knee reconstruction. Postoperative lateral (a) and anterioposterior (b) radiographs showing correct implant positioning. Due to the necessity of the patellar-ligament resection, absent soft tissue cover of the proximal anterior portion of the tibial implant caused wound-healing deficiencies, which required operative revisions (c). To achieve soft tissue coverage a medial gastrocnemius muscle flap was prepared (d) and covered with a mesh graft from the opposite thigh (e). 
Regrettably, after further progress towards mobility during his stationary rehabilitation, the patient deceased from an alveolitis. Post mortem examination to investigate the etiology of the lung disease was not performed. Therefore this case report is limited to the diagnostic and operative management, as well as the short-term outcome at discharge from our stationary care.

The comparison of outcomes with alternative surgical procedures (endoprosthetic reconstruction versus amputation) is hardly possible, due to the low incidence and different stages of the disease. To date, no clinical trial comparing outcomes of tumor replacing joint arthroplasty and amputation in joint-involving chondrosarcoma does exist to our knowledge. Therefore decision of surgical management is highly individual, primarily depending on disease stage, grade and patient preferences.

\section{Competing interests}

All authors declare that they have no competing interests.

\section{Authors' contributions}

JTS and AFS prepared the figures and collected the data. JTS, MR and AFS wrote the manuscript. All authors read and approved the final manuscript.

\section{Acknowledgement}

This publication was funded by the German Research Foundation (DFG) and the University of Wuerzburg in the funding programme Open Access Publishing.

Received: 16 November 2013 Accepted: 16 June 2014

Published: 24 June 2014

\section{References}

Aigner T (2002) Towards a new understanding and classification of chondrogenic neoplasias of the skeleton - biochemistry and cell biology of chondrosarcoma and its variants. Virchows Arch 441(3):219-230, doi:10.1007/s00428-002-0641-x

Dallas J, Imanirad I, Rajani R, Dagan R, Subbiah S, Gaa R, Dwarica WA, Ivey AM, Zlotecki RA, Malyapa R, Indelicato DJ, Scarborough MT, Reith JD, Gibbs CP, Dang LH (2012) Response to sunitinib in combination with proton beam radiation in a patient with chondrosarcoma: a case report. J Med Case Rep 6:41, doi:10.1186/1752-1947-6-41

Drilon AD, Popat S, Bhuchar G, D'Adamo DR, Keohan ML, Fisher C, Antonescu CR, Singer S, Brennan MF, Judson I, Maki RG (2008) Extraskeletal myxoid chondrosarcoma: a retrospective review from 2 referral centers emphasizing long-term outcomes with surgery and chemotherapy. Cancer 113(12):3364-3371, doi:10.1002/cncr.23978

Edge SB, Byrd DR, Compton CC, Fritz AG, Greene FL, Trotti A (2009) AJCC Cancer Staging Handbook: From the AJCC Cancer Staging Manual. Springer, Heidelberg

Giuffrida AY, Burgueno JE, Koniaris LG, Gutierrez JC, Duncan R, Scully SP (2009) Chondrosarcoma in the United States (1973 to 2003): an analysis of 2890 cases from the SEER database. J Bone Joint Surg Am 91(5):1063-1072, doi:10.2106/JBJS.H.00416

Goda JS, Ferguson PC, O'Sullivan B, Catton CN, Griffin AM, Wunder JS, Bell RS, Kandel RA, Chung PW (2011) High-risk extracranial chondrosarcoma. Cancer 117(11):2513-2519, doi:10.1002/cncr.25806

Han K, Sun Y-J, Shen Z, Zhang JJ, Lin F, Zhao H, Meerani S, Yao Y (2010) Extraskeletal myxoid chondrosarcoma: a case report of complete remission by chemotherapy and review of the literature. BMJ Case Rep 2010, doi:10.1136/bcr.07.2009.2128

Holzapfel B, Rechl H, Lehner S, Pilge H, Gollwitzer H, Steinhauser E (2011) Alloplastic reconstruction of the extensor mechanism after resection of tibial sarcoma. Sarcoma 2011, doi: 10.1155/2011/545104

Keel SB, Jaffe KA, Nielsen GP, Rosenberg AE (2001) Orthopaedic implant-related sarcoma: a study of twelve cases. Mod Pathol 14(10):969-977, doi:10.1038/ modpathol.3880420
Moussavi-Harami F, Mollano A, Martin JA, Ayoob A, Domann FE, Gitelis S, Buckwalter JA (2006) Intrinsic radiation resistance in human chondrosarcoma cells. Biochem Biophys Res Commun 346(2):379-385, doi:10.1016/j.bbrc.2006.05.158

Ofluoglu O, Boriani S, Gasbarrini A, De lure F, Donthineni R (2010) Diagnosis and planning in the management of musculoskeletal tumors: surgical perspective. Semin Intervent Radiol 27(2):185-190, doi:10.1055/s-0030-1253516

Onishi AC, Hincker AM, Lee FY (2011) Surmounting chemotherapy and radioresistance in chondrosarcoma: molecular mechanisms and therapeutic targets. Sarcoma 2011:1-8, doi:10.1155/2011/381564

Rutz HP, Weber DC, Goitein G, Ares C, Bolsi A, Lomax AJ, Pedroni E, Coray A, Hug EB, Timmermann B (2008) Postoperative spot-scanning proton radiation therapy for chordoma and chondrosarcoma in children and adolescents: initial experience at paul scherrer institute. Int J Radiat Oncol Biol Phys 71(1):220-225, doi:10.1016/j.jirobp.2007.09.014

Salis-Soglio G, Ghanem M, Meinecke I, Ellenrieder M, Klinger HM, Kirchhoff C (2010) The modular endoprothetic system Munich-Luebeck (MML): potential applications and results in lower extremities. Orthopaede 39(10):960-967, doi: 10.1007/s00132-009-1569-5

doi:10.1186/2193-1801-3-308

Cite this article as: Sieker et al:: Symptomatic loosening of a total knee arthroplasty caused by a tibial chondrosarcoma - a case report. SpringerPlus 2014 3:308.

\section{Submit your manuscript to a SpringerOpen ${ }^{\odot}$ journal and benefit from:}

- Convenient online submission

Rigorous peer review

- Immediate publication on acceptance

- Open access: articles freely available online

- High visibility within the field

- Retaining the copyright to your article

Submit your next manuscript at springeropen.com 